\title{
ON THE IMAGES OF ELLIPSES UNDER SIMILARITIES
}

\section{Nihal Yılmaz Özgür}

\begin{abstract}
We consider ellipses corresponding to any norm function on the complex plane and determine their images under the similarities which are special Möbius transformations.
\end{abstract}

\section{Introduction}

It is well-known that Möbius transformations map circles to circles where straight lines are considered to be circles through $\infty$. It is also well-known that all norms on $\mathbb{C}$ are equivalent. In [5], the present author considered circles corresponding to any norm function and determined their images under the Möbius transformations on the complex plane. Recently, in [2] and [3], Adam Coffman and Marc Frantz considered the images of non-circular ellipses (corresponding to the Euclidean norm function) under the Möbius transformations. In [6], the present author determined the images of non-circular ellipses under the harmonic Möbius transformations.

Motivated by the above studies, we consider the images of ellipses corresponding to any norm function on $\mathbb{C}$ under the Möbius transformations.

Key Words: Möbius transformation, ellipse, norm.

2010 Mathematics Subject Classification: Primary 30C35; Secondary 51F15.

Received: February, 2012.

Accepted: April, 2012. 
Throughout the paper, we consider the real linear space structure of the complex plane $\mathbb{C}$ and investigate the answer of the following question:

If $w=T(z)$ is a Möbius transformation and $\|\cdot\|$ is any norm function on $\mathbb{C}$, then does $T$ take ellipses to ellipses in this norm?

Note that all Möbius transformations do not map ellipses to ellipses corresponding to the Euclidean norm function on $\mathbb{C}$. From [2] and [3], we know that the Möbius transformations which map ellipses to ellipses are similarity transformations. In our case, we see that the rotation map $z \rightarrow e^{i \phi} z$ do not map ellipses to ellipses for every value of the real number $\phi$. Thus we restrict our investigations to similarity transformations.

\section{Main results}

We give a brief account of Möbius transformations (see [1] and [4] for more details).

A Möbius transformation $T$ is a function of the form

$$
T(z)=\frac{a z+b}{c z+d} ; a, b, c, d \in \mathbb{C} \text { and } a d-b c \neq 0 .
$$

Such transformations form a group under composition. The Möbius transformations with $c=0$ form the subgroup of similarities. Such transformations have the form

$$
z \rightarrow \alpha z+\beta ; \alpha, \beta \in \mathbb{C}, \alpha \neq 0 .
$$

The transformation $z \rightarrow \frac{1}{z}$ is called an inversion. Here we use the well-known fact that every Möbius transformation $T$ of the form (2.1) is a composition of finitely many similarities and inversions.

Let $\|$.$\| be any norm function on \mathbb{C}$. A circle whose center is at $z_{0}$ and of radius $r$ is denoted by $S_{r}\left(z_{0}\right)$ and defined by $S_{r}\left(z_{0}\right)=\left\{z \in \mathbb{C}:\left\|z-z_{0}\right\|=r\right\}$. An ellipse is the locus of points $z$ with the property that the sum of the distances from $z$ to two given fixed points, say $F_{1}$ and $F_{2}$, is a constant. The two fixed points are called foci. Thus the set $\left\{z \in \mathbb{C}:\left\|z-F_{1}\right\|+\left\|z-F_{2}\right\|=r\right\}$ is the ellipse with foci $F_{1}$ and $F_{2}$. We denote this ellipse by $E_{r}\left(F_{1}, F_{2}\right)$. If the two foci coincide, then the ellipse is a circle.

Now we recall the following lemma which will be used later. 
Lemma 2.1. [5] Let $\|$.$\| be any norm function on the complex plane. Then$ for every $\phi \in \mathbb{R}$, the following function define a norm on the complex plane:

$$
\|z\|_{\phi}=\left\|e^{-i \phi} z\right\| .
$$

We begin the following lemma.

Lemma 2.2. Let $\|$.$\| be any norm on \mathbb{C}$. Then the similarity transformations of the form

$$
f(z)=\alpha z+\beta ; \alpha \neq 0, \alpha \in \mathbb{R},
$$

map ellipses to ellipses corresponding to this norm function.

Proof. Let $\|$.$\| be any norm and let E_{r}\left(F_{1}, F_{2}\right)$ be any ellipse corresponding to this norm. If $f(z)$ is a similarity transformation of the form (2.4), then the image of $E_{r}\left(F_{1}, F_{2}\right)$ under $f$ is the ellipse $E_{|\alpha| r}\left(f\left(F_{1}\right), f\left(F_{2}\right)\right)$. Indeed, we have

$$
\begin{aligned}
& \left\|f(z)-f\left(F_{1}\right)\right\|+\left\|f(z)-f\left(F_{2}\right)\right\| \\
= & \left\|\alpha z+\beta-\left(\alpha F_{1}+\beta\right)\right\|+\left\|\alpha z+\beta-\left(\alpha F_{2}+\beta\right)\right\| \\
= & \left\|\alpha\left(z-F_{1}\right)\right\|+\left\|\alpha\left(z-F_{2}\right)\right\| \\
= & |\alpha|\left(\left\|z-F_{1}\right\|+\left\|z-F_{2}\right\|\right)=|\alpha| r .
\end{aligned}
$$

Now we consider the norm functions defined in (2.3). Notice that for the Euclidean norm, all of the norm functions $\|\cdot\|_{\phi}$ are equal to the Euclidean norm. For any other norm function we have $\|\cdot\|_{k \pi}=\|\cdot\|$ where $k \in \mathbb{Z}$.

Then we can give the following theorem:

Theorem 2.1. Let $w=f(z)=\alpha z+\beta ; \alpha \neq 0, \alpha, \beta \in \mathbb{C}$. Then for every ellipse $E_{r}\left(F_{1}, F_{2}\right)$ corresponding to any norm function $\|$.$\| on \mathbb{C}, f\left(E_{r}\left(F_{1}, F_{2}\right)\right)$ is an ellipse corresponding to the same norm function or corresponding to the norm function $\|z\|_{\phi}=\left\|e^{-i \phi} . z\right\|$, where $\phi=\arg (\alpha)$.

Proof. Let $w=f(z)=\alpha z+\beta ; \alpha \neq 0, \alpha, \beta \in \mathbb{C}$. If $E_{r}\left(F_{1}, F_{2}\right)$ is an Euclidean ellipse, then from [3] we know that $f\left(E_{r}\left(F_{1}, F_{2}\right)\right)$ is again an Euclidean ellipse. Suppose that $E_{r}\left(F_{1}, F_{2}\right)$ is not an Euclidean ellipse. Let us write $f(z)=$ 
$|\alpha| e^{i \phi} z+\beta ; \alpha \neq 0, \phi=\arg (\alpha)$ and let $f_{1}(z)=e^{i \phi} z, f_{2}(z)=|\alpha| z+e^{-i \phi} \beta$. We have $f(z)=\left(f_{1} \circ f_{2}\right)(z)$.

Then by Lemma 2.2 , the transformation $f_{2}(z)$ maps ellipses to ellipses corresponding to this norm function. Let $w=f_{1}(z)=e^{i \phi} z, \phi \neq k \pi, k \in \mathbb{Z}$. Now we consider the norm function $\|\cdot\|_{\phi}$ given in Lemma 2.1. We get

$$
\begin{aligned}
\left\|w-f\left(F_{1}\right)\right\|_{\phi}+\left\|w-f\left(F_{2}\right)\right\|_{\phi} & =\left\|e^{i \phi}\left(z-F_{1}\right)\right\|_{\phi}+\left\|e^{i \phi}\left(z-F_{2}\right)\right\|_{\phi} \\
& =\left\|e^{-i \phi}\left[e^{i \phi}\left(z-F_{1}\right)\right]\right\|+\left\|e^{-i \phi}\left[e^{i \phi}\left(z-F_{2}\right)\right]\right\| \\
& =\left\|z-F_{1}\right\|+\left\|z-F_{2}\right\|=r .
\end{aligned}
$$

This shows that the image of the ellipse $E_{r}\left(F_{1}, F_{2}\right)$ under the transformation $w=f_{1}(z)=e^{i \phi} z,(\phi \neq k \pi, k \in \mathbb{Z})$ is the ellipse $E_{r}\left(f\left(F_{1}\right), f\left(F_{2}\right)\right)$ corresponding to the norm function $\|\cdot\|_{\phi}$ given in (2.3).

We note that we do not know the exact values of $\phi$ for which $\|\cdot\|_{\phi}=\|\cdot\|$. This is an open problem. If $\|\cdot\|_{\phi}=\|\cdot\|$, then the transformation $f_{1}(z)=$ $e^{i \phi} z$ maps ellipses to ellipses corresponding to this norm function. In general $f_{1}(z)=e^{i \phi} z$ do not map ellipses to ellipses corresponding to the same norm function. For example, let $\|\cdot\|$ be any norm with $\|1\| \neq\|i\|$ and $\phi=\frac{\pi}{2}$. Assume that $\|z\|_{\frac{\pi}{2}}=\|z\|$ for all $z \in \mathbb{C}$. For $z=1$ we have $\|i\|=\|1\|$, which is a contradiction. Therefore the transformation $z \rightarrow e^{\frac{\pi}{2} i} z$ maps ellipses corresponding to the norm function $\|$.$\| to ellipses corresponding to the norm$ function $\|\cdot\|_{\frac{\pi}{2}}$. We give the following conjecture for the norm functions with the properties $\|1\|=\|i\|$ and $\|z\|=\|\bar{z}\|$ for all $z \in \mathbb{C}$.

Conjecture 2.1. Let $\|$.$\| be any norm on \mathbb{C}$ with $\|1\|=\|i\|$. Assume that $\|z\|=\|\bar{z}\|$ for all $z \in \mathbb{C}$. Then we have $\|\cdot\|_{\frac{\pi}{2}}=\|\cdot\|$ and hence the transformation $z \rightarrow e^{\frac{\pi}{2} i} z$ maps ellipses to ellipses corresponding to this norm function.

If this conjecture is true, then we have also the transformation $z \rightarrow e^{\frac{\pi}{2} i} z$ maps circles to circles corresponding to this norm function as a corollary.

Example 2.1. Let us consider the norm function

$$
\|z\|=2|x|+|y|
$$

on $\mathbb{C}$. Let $F_{1}=-1$ and $F_{2}=1$. The image of the ellipse $E_{6}\left(F_{1}, F_{2}\right)$ under the transformation $w=e^{\frac{\pi}{2} i} z$ is not an ellipse corresponding to the same norm but 


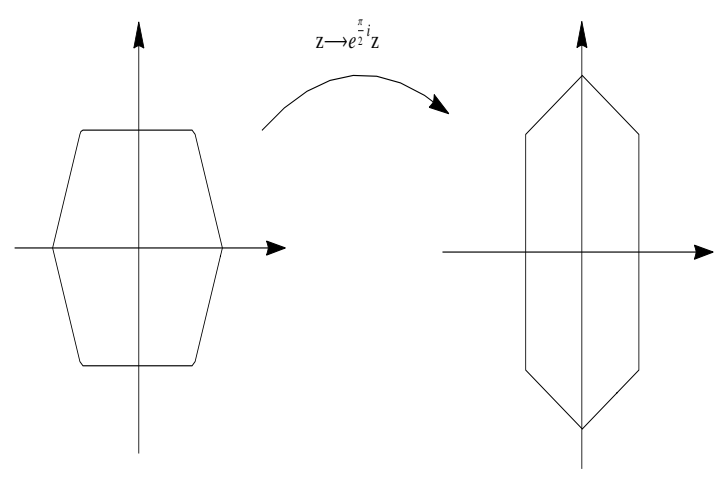

Figure 1:

it is the ellipse $E_{6}(-i, i)$ corresponding to the norm function $\|z\|_{\frac{\pi}{2}}=|x|+2|y|$, (see Figure 1).

Finally we note that Lemma 2.2 and Theorem 2.1 hold also for hyperbolas corresponding to any norm function on the complex plane.

\section{References}

[1] A. F. Beardon, Algebra and Geometry, Cambridge University Press, Cambridge, 2005. 
[2] A. Coffman and M. Frantz, Ellipses in the Inversive Plane, MAA Indiana Section Meeting, Mar. 2003.

[3] A. Coffman and M. Frantz, Möbius Transformations and Ellipses, The Pi Mu Epsilon Journal, 12 (2007), no.6, 339-345.

[4] G. A. Jones and D. Singerman, Complex functions. An algebraic and geometric viewpoint, Cambridge University Press, Cambridge, 1987.

[5] N. Yılmaz Özgür, On some mapping properties of Möbius transformations, Aust. J. Math. Anal. Appl., 6 (2009), no. 1, Art. 13, 8 pp.

[6] N. Yılmaz Özgür, Ellipses and Harmonic Möbius Transformations, An. Şt. Univ. Ovidius Constanta, 18 (2010), no. 2, 201-208.

Nihal YILMAZ ÖZGÜR,

Department of Mathematics,

Balıkesir University,

Balıkesir, Türkiye.

Email: nihal@balikesir.edu.tr 